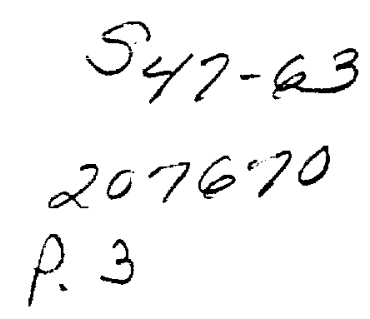

\author{
6068 \\ Graphical Simulation for \\ Aerospace Manufacturing \\ AIAA-94-1224-CP \\ Majid Babai
NASA, Marshall Space Center, AL. \\ Christopher Bien \\ Deneb Robotics, Inc., Auburn Hills, MI.
}

\begin{abstract}
$\underline{\text { Abstract }}$
Simulation software has become a key technological enabler for integrating flexible manufacturing systems and streamlining the overall aerospace manufacturing process. In particular, robot simulation and offline programming software is being credited for reducing down time and labor cost, while boosting quality and significantly increasing productivity.
\end{abstract}

\section{Graphical Simulation}

Simulation technology enables aerospace engineers to capture and evaluate a comprehensive robotic workcell proposal, at a single focal point. This type of functionality, found only in simulation, creates autonomy and facilitates progressive design methods such as concurrent engineering. Used as a concurrent engineering tool real-time simulation of geometrically accurate robots, tools, and peripheral components enables seamless communication between various parties such as design engineers, plant floor engineers, management, vendors, safety engineers, integrators, machine operators, and customers. In addition, many companies are using such tools to create 3-D animated proposals. They are quickly and easily creating simulations of their designs and manufacturing concepts. Contrary to the typical scenario of a customer laboring over a 1200 page proposal comprised of 2-D drawings, charts and descriptions, clients can now interactively preview an accurate simulation of their desired manufacturing process. Ultimately, simulation instills a high level of confidence, understanding, and realistic expectations in the potential customer.
Sophisticated continuous-event simulation technology enables the modeling of geometrically precise and accurately calibrated robots and entire workcells for analyzing every possible scenario. Extremely accurate simulations are possible by incorporating the actual robot attributes such as motion planning, kinematics, dynamics, and I/O logic.

Another advantage that simulation technology has over traditional prototyping methodologies is the ability to save complete libraries of robots, robot accessories, human models, cycle times, and entire workcells on a few megabytes of disc space for future reference or modification. The ability to store and retrieve simulation workcells enables engineers to reuse previously modeled parts, tooling, end effectors, robots, and processes. Simulation encourages "What if...?" experiments and assists engineers in making well informed, confident decisions.

Using these capabilities, NASA has developed a new system for removing the thermal insulation from the space shuttle's Solid Rocket Boosters (SRB) during disassembly at Kennedy Space Center. The thermal insulation is removed from the boosters by high-pressure waterjets, after which the motor segments are separated and sent to the Thiokol manufacturing plant in Utah for refurbishment and reuse. Previously, high pressure stripping nozzles were mounted onto a hand-held gun operated by a technician wearing a bulky protection suit. In order to remove the operator from a hazardous location, and enhance the process, the stripping nozzles were mounted onto a GMF S-420 robot on a mobile carrydeck. The boosters are cylindrical in shape and approximately $149 \mathrm{ft}$ long, with a diameter of $12 \mathrm{ft}$, consisting of four motor case segments, an aft skirt, and a forward skirt with a frustum containing parachutes. 


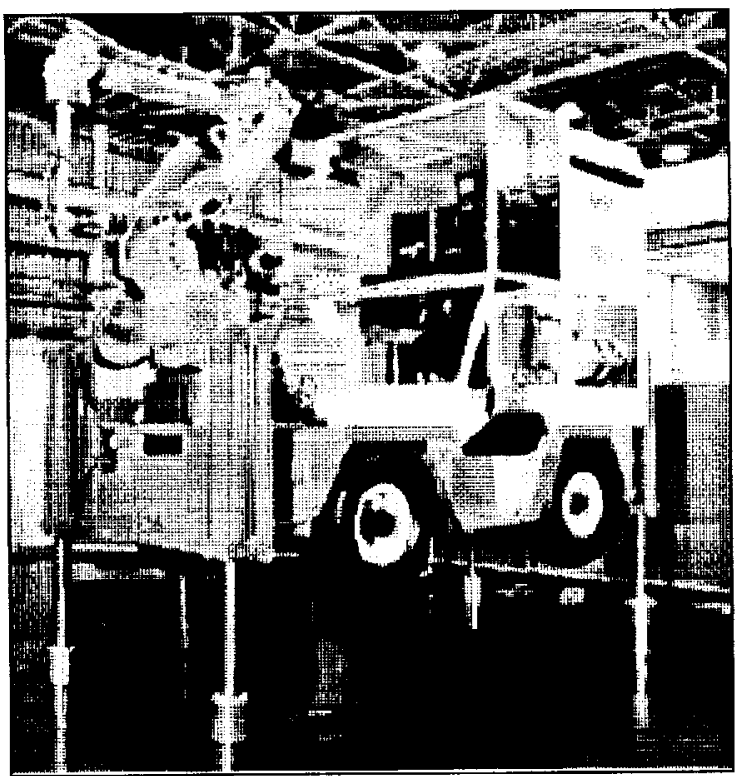

GMF S-420 robot on mobile carrydeck.

By utilizing IGRIP, robot simulation and off-line programming software from Deneb Robotics, Inc. NASA and USBI (United Space Boosters, Inc.) engineers at the Marshall Space Flight Center created a graphical workcell model of the robotic system and the facility. For modeling complex b-spline surfaces, the engineers first used Intergraph's Engineering Modeling System (I/EMS) to create solid models, which were then directly translated into IGRIP-format polygons for simulation.

NASA was faced with a unique situation. The robot's paths were programmed using the GMF Karel language and could not be tested off-line, due to the expense of creating an accurate full-size model of the boosters. To compound the problem further, the timeline set for disassembly processing (designed to prevent corrosion of the solid rocket motor's steel casing) and expediting SRB turnaround did not allow for extensive development testing to validate the robot paths. Using computer-based solid models and IGRIP simulation software, it was possible to choose the S-420 robot from the robot library and develop accurate stripping paths on an engineering workstation.

Simulation is instrumental for solving process optimization issues, which include robot motion planning, cycle time prediction, collision detection, and off-line programming.
Robot motion planning was once a speculative risk. During the pre-simulation era, process engineers relied primarily on their years of experience and rules of thumb to estimate critical factors such as the robot speed, joint values, and TCP (tool center point) path. In contrast with past methods, simulation is a powerful decision making tool for determining exact robot motion calculations. By incorporating such elements as inverse kinematics, dynamics, and robot I/O signals into a robot simulation workcell, robot motions can be computed and evaluated with great confidence.

Cycle time is another important aspect of process optimization. Minimizing overall cycle time directly translates into dollars saved. Through real-time simulation, NASA was able to predict run-time lengths of each stripping cycle accurately, before setting up on the plant floor. Here simulation shows its true mettle as an interactive tool for engineering analysis. Questions which once plagued manufacturing engineers such as, "Am I running my machines at the optimal cycle time? Can I speed up my cycle time?" or, "Do I need additional robots?" are resolved through the power of simulation.

Robot collisions are the number-one factor for expensive labor, tooling and downtime costs. The probability of collisions has been dramatically reduced through implementing accurate surface-to-surface collision and near-miss detection and exact minimumdistance calculations found in advanced robot workcell simulation software packages. Collision detection is an ideal engineering tool for identifying the "unexpected" hazards associated with tight tolerances, complex articulated robot motion, and human error.

Collision detection was a serious concern for the NASA team. The boosters contain a pyrotechnic linear shaped charge that is part of the range safety system which is designed to destroy the boosters in the event of a malfunction. To access this linear shaped charge, cork insulation has to be tripped from the system's tunnel covers. Additionally, the robot arm needs to work close to the Thrust Vector Control (TVC) system which power two hydraulic actuators that gimbal the nozzles. Hazardous hypergolic fuel is used to power the hydraulic subsystem; therefore, any leak caused by a collision would endanger personnel. Here, simulation helped to increase confidence and reduce the design cycle for the robot path before actual testing began. 


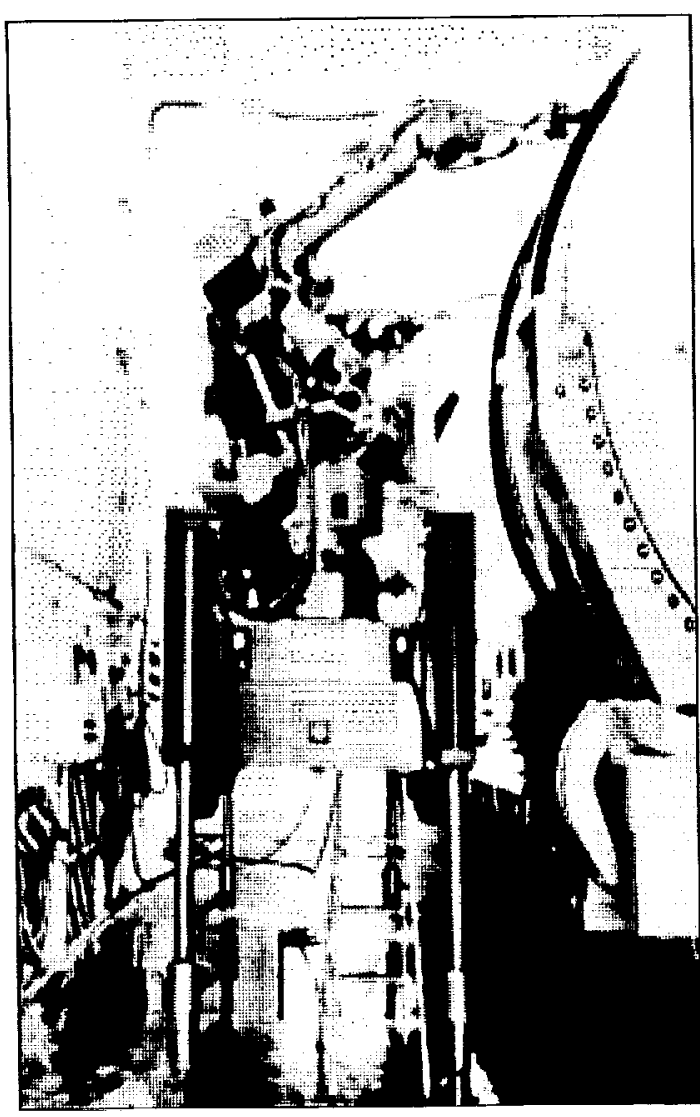

Due to the limited physical workspace, collisions were a serious concern for the NASA team.

Uniquely functional calibration enhancements have made simulation a practical tool for all aspects of automation. User friendly, proven calibration tools are applied to transform "picture perfect" simulation workcells into real-world parameters. By using calibration techniques to mimic the actual world environment, off-line programming has become reality.

The power of simulation and off-line programming is two-fold. First, the robot program is developed and verified in the simulation environment. Second, this program can be translated and downloaded to a specific manufacturer's controller for final touchup. As a result, off-line programming takes a fraction of the time allotted for conventional on-line programming practices.

In the aerospace manufacturing environment, in which product geometry is complex, startup time is limited, model changes are frequent, or the end products are large, off-line programming is the best answer for complex automated production lines and rapid responses to product/process changes. For NASA, simulation capabilities allow engineering responsibilities to be met when configurations on the flight hardware or in the workcell take place. 